PROCEEDINGS OF THE

AMERICAN MATHEMATICAL SOCIETY

Volume 136, Number 12, December 2008, Pages 4437-4444

S 0002-9939(08)09497-5

Article electronically published on July 30, 2008

\title{
A SUMMABILITY CRITERION FOR STOCHASTIC INTEGRATION
}

\author{
NICOLAE DINCULEANU AND PETER GRAY
}

(Communicated by Richard C. Bradley)

\begin{abstract}
In this paper we give simple, sufficient conditions for the existence of the stochastic integral for vector-valued processes $X$ with values in a Banach space $E$; namely, $X$ is of class (LD), and the stochastic measure $I_{X}$ is bounded and strongly additive in $L_{E}^{p}$ (in particular, if $I_{X}$ is bounded in $L_{E}^{p}$ and $c_{0} \nsubseteq E$ ) and has bounded semivariation. The result is then applied to martingales and processes with integrable variation or semivariation. For martingales the condition of being of class (LD) is superfluous. For a square-integrable martingale with values in a Hilbert space, all the conditions are superfluous. For processes with $p$-integrable semivariation or $p$-integrable variation, the conditions of $I_{X}$ to be bounded and have bounded semivariation are superfluous. For processes with 1-integrable variation, all conditions are superfluous. In a forthcoming paper, we shall extend these results to local summability. The extension needs additional nontrivial work.
\end{abstract}

\section{PRELIMINARIES}

We shall use the following definitions and notation (cf. [D-M] and [D]).

1) $(\Omega, \mathcal{F}, P)$ is a probability space and $\left(\mathcal{F}_{t}\right)_{t \in \mathbb{R}_{+}}$is a filtration satisfying the usual conditions; i.e., $\mathcal{F}_{s} \subset \mathcal{F}_{t}$ if $s<t$, and $\mathcal{F}_{t}=\bigcap_{s>t} \mathcal{F}_{s}$, for $t \in \mathbb{R}$.

$\mathcal{R}$ is the ring generated by the semiring of predictable rectangles of the form $\{0\} \times A$ with $A \in \mathcal{F}_{0}$ and $(s, t] \times A$ with $s, t \in \mathbb{R}_{+}$and $A \in \mathcal{F}_{s}$.

$\mathcal{P}$ is the $\sigma$-algebra of predictable sets, or the predictable $\sigma$-algebra, and is generated by the ring $\mathcal{R}$.

2) $E, F, G$ are Banach spaces such that $E \subset L(F, G)$ continuously; i.e.,

$$
|x y| \leq|x||y| \text {, for } x \in E \text { and } y \in F \text {. }
$$

$L_{E}^{p}$ will denote the space $L_{E}^{p}(P), 1 \leq p \leq+\infty$, and $L^{p}$ will denote $L_{\mathbb{R}}^{p}$.

3) $X: \mathbb{R}_{+} \times \Omega \longrightarrow E$ is a cadlag, adapted process and $1 \leq p<\infty$.

We assume that $X_{t} \in L_{E}^{p}$ for every $t \in \mathbb{R}_{+}$. We say $X$ is of class (D) if the set of random variables $\left\{X_{T} \mathbf{1}_{\{T<\infty\}} ; T\right.$ a stopping time $\}$ is uniformly integrable (cf. [D-M, Definition VI.20]). We say $X$ is of class (LD) if for every $a \in \mathbb{R}$, the set of random variables $\left\{X_{T} ; T: \Omega \longrightarrow[0, a]\right.$ a stopping time $\}$ is uniformly integrable

Received by the editors May 3, 2007, and, in revised form, June 7, 2007, and November 23, 2007.

2000 Mathematics Subject Classification. Primary 60G20; Secondary 60G44.

Key words and phrases. Stochastic processes, Stochastic integral, summable processes.

(C)2008 American Mathematical Society 
([M, Definition 13.1]). We define the additive measure $I_{X}: \mathcal{R} \longrightarrow L_{E}^{p}$ first for the predictable rectangles by

$$
I_{X}(\{0\} \times A)=X_{0} \mathbf{1}_{A}, \text { for } A \in \mathcal{F}_{0},
$$

and

$$
I_{X}((s, t] \times A)=\left(X_{t}-X_{s}\right) \mathbf{1}_{A}, \text { for } A \in \mathcal{F}_{s},
$$

and then by additivity, for the whole ring $\mathcal{R}$.

We say that the process $X$ is p-summable relative to $\left(F, L_{G}^{p}\right)$ if the measure $I_{X}$ can be extended to a $\sigma$-additive measure $I_{X}: \mathcal{P} \longrightarrow L_{E}^{p}$ with bounded semivariation relative to $\left(F, L_{G}^{p}\right)$. (See $[\mathrm{D}, \S 4$, for the definition and the properties of the semivariation.) If $X$ is $p$-summable, we can define the stochastic integral $H \cdot X$ for certain predictable processes $H: \mathbb{R}_{+} \times \Omega \longrightarrow F$.

The stochastic integral $H \cdot X$ is itself a process, and we have $(H \cdot X)_{t} \in L_{G}^{p}$ for $t \in \mathbb{R}_{+}$(see [D, Chapter 2]).

4) A natural problem is to give simple, sufficient conditions for the summability of $X$. We show in this paper that if $X$ is of class (LD) and if $I_{X}$ is bounded and strongly additive (in particular, if $I_{X}$ is bounded and $c_{0} \nsubseteq E E$ ), then $X$ is $p$-summable relative to $\left(\mathbb{R}, L_{G}^{p}\right)$. If $I_{X}$ also has bounded semivariation relative to $\left(F, L_{G}^{p}\right)$, then $X$ is $p$-summable relative to $\left(F, L_{G}^{p}\right)$ (Theorem 3$)$. We apply this summability criterion to martingales (Theorems 4 and 5 ), to processes with integrable semivariation (Theorem 6) and to processes with integrable variation (Theorems 7 and 8). For martingales, the condition of class (LD) is automatically satisfied. For processes with $p$-integrable semivariation, if the embedding $E \subset L(F, G)$ is an isometry, then $I_{X}$ is automatically bounded and has bounded semivariation relative to $\left(F, L_{G}^{p}\right)$. For processes with $p$-integrable variation, the isometry of $E \subset L(F, G)$ can be removed. Further, for $p=1$, even the condition $c_{0} \nsubseteq E$ can be removed.

\section{EXTENSION OF THE MEASURE $I_{X}$}

The main part of the summability criterion is the extension of the measure $I_{X}$. This is stated in terms of strong additivity of $I_{X}$. A measure $m: \mathcal{R} \longrightarrow E$ defined on a ring $\mathcal{R}$ is said to be strongly additive if for any sequence $\left(A_{n}\right)$ of disjoint sets from $\mathcal{R}$ we have $m\left(A_{n}\right) \longrightarrow 0$. If $m$ is bounded and $c_{0} \nsubseteq E E$, then $m$ is strongly additive ([D, Theorem 6.8]). A $\sigma$-additive measure on a $\sigma$-ring is strongly additive, but the converse is not necessarily true. For a converse property, see the extension Theorem 7.7 in [D]. For the properties of a strongly additive measure, see [D], $\S 6$.

Theorem 1. Let $X: \mathbb{R}_{+} \times \Omega \longrightarrow E$ be a cadlag, adapted process such that $X_{t} \in L_{E}^{p}$ for every $t \in \mathbb{R}_{+}$. Assume that $X$ is of class $(L D)$, and that $I_{X}$ is bounded and strongly additive in $L_{E}^{p}$ on $\mathcal{R}$. Then $I_{X}$ can be extended to a $\sigma$-additive measure $I_{X}: \mathcal{P} \longrightarrow L_{E}^{p}$.

Proof. Let $Z=L_{E^{*}}^{\infty} \subset L_{E^{*}}^{q}, \frac{1}{p}+\frac{1}{q}=1$. Then $Z$ is a norming space for $L_{E}^{p}$. Let $g \in Z$. Regard the real-valued measure $\left\langle I_{X}, g\right\rangle: \mathcal{R} \longrightarrow \mathbb{R}$ defined by

$$
\left\langle I_{X}, g\right\rangle(B)=\left\langle I_{X}(B), g\right\rangle=\int\left\langle I_{X}(B), g\right\rangle d P \text { for } B \in \mathcal{R},
$$

where the second bracket represents the duality between $L_{E}^{p}$ and $L_{E^{*}}^{q}$ and the bracket in the integral represents the duality between $E$ and $E^{*}$. For each $B \in \mathcal{R}$ we have

$$
\left|\left\langle I_{X}, g\right\rangle(B)\right| \leq\left\|I_{X}(B)\right\|_{p}\|g\|_{q} ;
$$


hence $\left\langle I_{X}, g\right\rangle$ is bounded on $\mathcal{R}$. Since $\left\langle I_{X}, g\right\rangle$ is real-valued, it has bounded variation on $\mathcal{R}$ ( $[\mathrm{D}$, Proposition 2.16b]). Denote by $G$ the cadlag version of the martingale $\left(E\left[g \mid \mathcal{F}_{t}\right]\right)_{t \in \mathbb{R}_{+}}$. Henceforth, the context will determine whether we are referring to the Banach space $G$ or the martingale $G$. We have

$$
G_{t}=E\left[g \mid \mathcal{F}_{t}\right] \text { a.s. }
$$

and

$$
\left|G_{t}\right| \leq E\left[|g| \mid \mathcal{F}_{t}\right] \leq\|g\|_{\infty} \text { a.s. }
$$

Consider the real-valued process $X G$ defined by $(X G)_{t}=\left\langle X_{t}, G_{t}\right\rangle$ for $t \in \mathbb{R}_{+}$, where the bracket represents the duality between $E$ and $E^{*}$. The Dooléans measure $\mu_{X G}$ of $X G$ is defined for predictable rectangles by

$$
\mu_{X G}(\{0\} \times A)=E\left[X_{0} G_{0} \mathbf{1}_{A}\right], \text { for } A \in \mathcal{F}_{0},
$$

and

$$
\mu_{X G}((s, t] \times A)=E\left[\left(X_{t} G_{t}-X_{s} G_{s}\right) \mathbf{1}_{A}\right], \text { for } A \in \mathcal{F}_{s}
$$

and then by additivity on the whole ring $\mathcal{R}$.

We have $\mu_{X G}=\left\langle I_{X}, g\right\rangle$. In fact, if $B=\{0\} \times A$ with $A \in \mathcal{F}_{0}$, then

$$
\begin{aligned}
\left\langle I_{X}, g\right\rangle(B) & =\int\left\langle I_{X}(B), g\right\rangle d P=\int_{A}\left\langle X_{0}, g\right\rangle d P \\
& =\int_{A}\left\langle X_{0}, G_{0}\right\rangle d P=\int_{A}(X G)_{0} \mathbf{1}_{A} d P=\mu_{X G}(\{0\} \times A)=\mu_{X G}(B) .
\end{aligned}
$$

If $B=(s, t] \times A$ with $A \in \mathcal{F}_{s}$, then

$$
\begin{aligned}
\left\langle I_{X}, g\right\rangle(B)=\int_{A}\left\langle X_{t}-X_{s}, g\right\rangle d P & =\int_{A}\left\langle X_{t}, G_{t}\right\rangle d P \\
& -\int_{A}\left\langle X_{s}, G_{s}\right\rangle d P=\mu_{X G}((s, t] \times A)=\mu_{X G}(B) .
\end{aligned}
$$

Then by additivity, we have

$$
\left\langle I_{X}, g\right\rangle(B)=\mu_{X G}(B) \text { for } B \in \mathcal{R} .
$$

Since $I_{X}$ is bounded on $\mathcal{R}$ by hypothesis, it follows that $\mu_{X G}$ is bounded; hence it has bounded variation on $\mathcal{R}$. This means that $X G$ is a quasimartingale (M, Definition 8.6]). Moreover, $X G$ is a quasimartimgale of class (LD) ([M, Definition 13.1]). In fact, let $a \in \mathbb{R}_{+}$and let $T: \Omega \longrightarrow[0, a]$ be a simple stopping time. Then

$$
\left|(X G)_{T}\right|=\left|\left\langle X_{T}, G_{T}\right\rangle\right| \leq\left|X_{T}\left\|G_{T}|\leq| X_{T} \mid\right\| g \|_{\infty} .\right.
$$

Since, by hypothesis, $X$ is of class (D) on $[0, a]$, it follows that $X G$ is of class (D) on $[0, a]$; and since $a \in \mathbb{R}_{+}$was arbitrary, it follows that $X G$ is of class (LD). We can then apply Theorem 13.3 in $\mathrm{M}$ and deduce that $\mu_{X G}$ can be extended to a $\sigma$-additive measure $\mu_{X G}: \mathcal{P} \longrightarrow \mathbb{R}$; that is, the measure $\left\langle I_{X}, g\right\rangle$ can be extended to a $\sigma$-additive measure on $\mathcal{P}$. Since $I_{X}$ is strongly additive, we can then apply the extension Theorem 7.7 in $\left[\mathrm{D}\right.$, and deduce that $I_{X}$ can be extended to a $\sigma$-additive measure $I_{X}: \mathcal{P} \longrightarrow L_{E}^{p}$.

Corollary 2. Let $X: \mathbb{R}_{+} \times \Omega \longrightarrow E$ be a cadlag, adapted process such that $X_{t} \in L_{E}^{p}$ for every $t \in \mathbb{R}_{+}$. Assume that $c_{0} \nsubseteq E, X$ is of class $(L D)$ and $I_{X}$ is bounded in $L_{E}^{p}$ on $\mathcal{R}$. Then $I_{X}$ can be extended to a $\sigma$-additive measure $I_{X}: \mathcal{P} \longrightarrow L_{E}^{p}$. 
Proof. In fact, if $c_{0} \nsubseteq E E$ and $I_{X}$ is bounded in $L_{E}^{p}$ on $\mathcal{R}$, then $I_{X}$ is strongly additive in $L_{E}^{p}$ on $\mathcal{R}([\mathrm{D}$, Theorem 6.8]).

\section{THE SUMMABILITY CRITERION}

We can now state the summability criterion.

Theorem 3. Let $X: \mathbb{R}_{+} \times \Omega \longrightarrow E$ be a cadlag, adapted process such that $X_{t} \in L_{E}^{p}$ for every $t \in \mathbb{R}_{+}$. Assume that $X$ is of class (LD) and $I_{X}$ is bounded and strongly additive in $L_{E}^{p}$ on $\mathcal{R}$. (In particular, this is true if $X$ is of class $(L D), c_{0} \nsubseteq E$, and $I_{X}$ is bounded in $L_{E}^{p}$ on $\mathcal{R}$.)

a) If we consider $E=L(\mathbb{R}, E)$, hence $L_{E}^{p}=L\left(F, L_{G}^{p}\right)$, then $X$ is p-summable relative to $\left(\mathbb{R}, L_{E}^{p}\right)$.

b) If $E \subset L(F, G)$, hence $L_{E}^{p} \subset L\left(F, L_{G}^{p}\right)$, and if $I_{X}$ has bounded semivariation on $\mathcal{R}$ relative to $\left(F, L_{G}^{p}\right)$, then $X$ is p-summable relative to $\left(F, L_{G}^{p}\right)$.

Proof. By Theorem 1 and Corollary 2, $I_{X}$ can be extended to a $\sigma$-additive measure $I_{X}: \mathcal{P} \longrightarrow L_{E}^{p}$. Then $I_{X}$ is bounded on $\mathcal{P}\left(\left[\mathbb{D}\right.\right.$, Theorem 2.14]) and $I_{X}$ has bounded semi-variation on $\mathcal{P}$ relative to $\left(\mathbb{R}, L_{E}^{p}\right)$ ([D, Proposition 4.14]). It follows that $X$ is $p$-summable relative to $\left(\mathbb{R}, L_{E}^{p}\right)$, which proves assertion a).

If $L_{E}^{p} \subset L\left(F, L_{G}^{p}\right)$ and if $I_{X}$ has bounded semivariation on $\mathcal{R}$ relative to $\left(F, L_{G}^{p}\right)$, then $I_{X}$ has bounded semivariation on $\mathcal{P}$ relative to $\left(F, L_{G}^{p}\right)$ ([D, Corollary 7.6]). It follows that $X$ is $p$-summable relative to $\left(F, L_{G}^{p}\right)$, which proves assertion b).

\section{Summability OF MARTINGALES}

For martingales the condition of being of class (LD) is automatically satisfied. We then have the following theorem.

Theorem 4. Let $X: \mathbb{R}_{+} \times \Omega \longrightarrow E$ be a right continuous martingale such that $X_{t} \in L_{E}^{p}$ for every $t \in \mathbb{R}_{+}$. Assume $c_{0} \nsubseteq E$.

a) If $I_{X}$ is bounded in $L_{E}^{p}$ on $\mathcal{R}$, then $X$ is p-summable relative to $\left(\mathbb{R}, L_{E}^{p}\right)$.

b) If $I_{X}$ is bounded in $L_{E}^{p}$ on $\mathcal{R}$ and has bounded semivariation on $\mathcal{R}$ relative to $\left(F, L_{G}^{p}\right)$, then $X$ is p-summable relative to $\left(F, L_{G}^{p}\right)$.

For square-integrable martingales in Hilbert spaces, all the conditions of Theorem 3 are satisfied, and we have the following theorem.

Theorem 5. Let $X: \mathbb{R}_{+} \times \Omega \longrightarrow E \subset L(F, G)$ be a square-integrable martingale such that both $E$ and $G$ are Hilbert spaces. Then $X$ is a 2-summable process relative to $L\left(F, L_{G}^{2}\right)$.

Proof. Since $E$ is a Hilbert space, we have $c_{0} \not E E$. Since $X$ is a square-integrable martingale, it is of class (D). Finally, $I_{X}$ is bounded in $L_{E}^{2}$ on $\mathcal{R}$ (see the proof of Lemma 17.1 and Theorem 17.2 in $\left[\mathrm{D}\right.$ ), and $I_{X}$ has bounded semivariation on $\mathcal{R}$ relative to $L\left(F, L_{G}^{2}\right)$ (see [D, Theorem 17.5]). By Theorem $4, X$ is 2 -summable relative to $L\left(F, L_{G}^{2}\right)$.

\section{SumMability OF PROCESSES WITH INTEGRABLE VARIATION OR INTEGRABLE SEMIVARIATION}

For the definition and properties of processes with integrable variation or integrable semivariation, we refer to [D, $\S \S 19$ and 21]. For the Lebesgue-Stieltjes 
integral with respect to functions with finite variation or finite semivariation, see D, $\S 18 \mathrm{D}$ and $\S 20 \mathrm{D}]$.

For a cadlag, adapted process $X: \mathbb{R}_{+} \times \Omega \longrightarrow E \subset L(F, G)$, we denote by $|X|\left(\right.$ resp. $\left.\widetilde{X}_{F, G}\right)$ the variation process (resp. the semivariation process relative to $(F, G))$ of $X$.

We say that $X$ has $p$-integrable variation (resp. p-integrable semivariation relative to $(F, G))$ if the total variation $|X|_{\infty}$ (resp. the total semivariation $\left.\left(\widetilde{X}_{F, G}\right)_{\infty}\right)$ belongs to $L^{p}$.

We state first the summability property of processes with integrable semivariation.

Theorem 6. Let $X: \mathbb{R}_{+} \times \Omega \longrightarrow E \subset L(F, G)$ be a cadlag, adapted process. Assume that the embedding $E \subset L(F, G)$ is an isometry, that $c_{0} \nsubseteq E$, and that $X$ has p-integrable variation or p-integrable semivariation relative to $L\left(F, L_{G}^{p}\right)$. Then $X$ is p-summable relative to $L\left(F, L_{G}^{p}\right)$.

Proof. If $X$ has $p$-integrable variation, then it has $p$-integrable semivariation relative to $(F, G)$. Therefore, we shall give the proof on the assumption that $X$ has $p$ integrable semivariation relative to $(F, G)$. Since the embedding $E \subset L(F, G)$ is an isometry, we have (see [D, Property 4.3])

$$
\left|X_{t}\right| \leq\left(\widetilde{X}_{F, G}\right)_{t} \leq\left(\widetilde{X}_{F, G}\right)_{\infty} \in L^{p}
$$

therefore, $X_{t} \in L^{p}$ for every $t \in \mathbb{R}_{+}$. Then, for every stopping time $T$ we have

$$
\left|X_{T}\right| \leq\left(\widetilde{X}_{F, G}\right)_{\infty}
$$

hence $X$ is of class (D). It remains to prove that $I_{X}$ has bounded semivariation relative to $L\left(F, L_{G}^{p}\right)$. It will follow then that $I_{X}$ is bounded in $L_{E}^{p}$. We have

$$
I_{X}(B)(\varpi)=\int \mathbf{1}_{B}(s, \varpi) d X_{s}(\varpi), \text { for } B \in \mathcal{R},
$$

where, for each $\varpi \in \Omega$, we used the Lebesgue-Stieltjes integral with respect to the function $s \longmapsto X_{s}(\varpi)$ with bounded semivariation relative to $(F, G)$.

For $B=\{0\} \times A$ with $A \in \mathcal{F}_{0}$ we have

$$
I_{X}(B)(\varpi)=\mathbf{1}_{A}(\varpi) X_{0}(\varpi)=\int \mathbf{1}_{B}(s, \varpi) d X_{s}(\varpi),
$$

and for $B=(u, v] \times A$ with $A \in \mathcal{F}_{u}$, we have

$$
\begin{gathered}
I_{X}(B)(\varpi)=\mathbf{1}_{A}(\varpi)\left(X_{v}(\varpi)-X_{u}(\varpi)\right) \\
=\mathbf{1}_{A}(\varpi) \int \mathbf{1}_{(u, v]}(s, \varpi) d X_{s}(\varpi)=\int \mathbf{1}_{B}(s, \varpi) d X_{s}(\varpi) .
\end{gathered}
$$

Then the equality remains valid for every $B \in \mathcal{R}$.

Let $B_{1}, \ldots, B_{n}$ be disjoint sets from $\mathcal{R}$ and $x_{1}, \ldots, x_{n}$ elements from $F$ with $\left|x_{i}\right|$ $\leq 1$ for $1 \leq i \leq n$. Without loss of generality, we can assume that each $B_{i}$ is of the form $B_{i}=\left(u_{i}, v_{i}\right] \times A_{i}$ with $A_{i} \in \mathcal{F}_{u_{i}}$ and that $u_{1}<v_{1} \leq u_{2}<v_{2} \leq \ldots \leq u_{n}<v_{n}$. 
For each $\varpi \in \Omega$ we have then, taking the supremum for all $z \in G^{*}$ with $|z| \leq 1$,

$$
\begin{gathered}
\left|\sum_{i} I_{X}\left(B_{i}\right)(\varpi) x_{i}\right|=\left|\sum_{i} \mathbf{1}_{A_{i}}(\varpi)\left(X_{v_{i}}(\varpi)-X_{u_{i}}(\varpi)\right) x_{i}\right| \\
=\sup \left|\sum_{i} \mathbf{1}_{A_{i}}(\varpi)\left\langle\left(X_{v_{i}}(\varpi)-X_{u_{i}}(\varpi)\right) x_{i}, z\right\rangle\right| \\
\leq \sup \sum_{i} \mathbf{1}_{A_{i}}(\varpi)\left|\left\langle X_{v_{i}}(\varpi) x_{i}, z\right\rangle-\left\langle X_{u_{i}}(\varpi) x_{i}, z\right\rangle\right| \\
\leq \sup \sum_{i}\left|\left(\left(X_{z}\right)_{v_{i}}(\varpi)-\left(X_{z}\right)_{u_{i}}(\varpi)\right) x_{i}\right| \\
\leq \sup \sum_{i}\left|\left(X_{z}\right)_{v_{i}}(\varpi)-\left(X_{z}\right)_{u_{i}}(\varpi)\right| \leq \sup \left|X_{z}\right|_{\infty}(\varpi)=\left(\widetilde{X}_{F, G}\right)_{\infty}(\varpi) .
\end{gathered}
$$

We used Proposition 20.7 in [D] to compute the semivariation $\widetilde{X}_{F, G}$ by means of the variation $\left|X_{z}\right|$. Then

$$
\left\|\sum_{i} I_{X}\left(B_{i}\right) x_{i}\right\|_{p} \leq\left\|\left(\widetilde{X}_{F, G}\right)_{\infty}\right\|_{p} .
$$

Taking the supremum for all $B_{i}$ and $x_{i}$ as above we get

$$
\left(\widetilde{I_{X}}\right)_{F, L_{G}^{p}}\left(\mathbb{R}_{+} \times \Omega\right) \leq\left\|\left(\widetilde{X}_{F, G}\right)_{\infty}\right\|_{p}<\infty ;
$$

i.e., $I_{X}$ has bounded semivariation relative to $\left(F, L_{G}^{p}\right)$.

In the above set of inequalities, taking just a set $B$ in $\mathcal{R}$ and an element $x$ in $F$ with $|x| \leq 1$, we get

$$
\left|I_{X}(B)(\varpi) x\right| \leq\left(\widetilde{X}_{F, G}\right)_{\infty}(\varpi) .
$$

Since the embedding $E \subset L(F, G)$ is an isometry, taking the supremum for $x$ in $F$ with $|x| \leq 1$ we have

therefore

$$
\left|I_{X}(B)(\varpi)\right| \leq\left(\widetilde{X}_{F, G}\right)_{\infty}(\varpi) ;
$$

$$
\left\|I_{X}(B)\right\|_{p} \leq\left\|\left(\widetilde{X}_{F, G}\right)_{\infty}\right\|_{p} .
$$

Then $I_{X}$ is bounded in $L_{E}^{p}$ on $\mathcal{R}$. By the Summability Criterion, Theorem 3 , it follows that $X$ is $p$-summable relative to $\left(F, L_{G}^{p}\right)$.

Remark. This is a new proof of Theorem 21.12a in [D].

If $X$ has $p$-integrable variation, we can remove the condition that the embedding $E \subset L(F, G)$ is an isometry.

Theorem 7. Let $X: \mathbb{R}_{+} \times \Omega \longrightarrow E \subset L(F, G)$ be a cadlag, adapted process. Assume that $c_{0} \nsubseteq E$ and that $X$ has p-integrable variation. Then, for any embedding $E \subset L(F, G), X$ is p-summable relative to $L\left(F, L_{G}^{p}\right)$.

Proof. For every $t \in \mathbb{R}_{+}$we have

$$
\left|X_{t}\right| \leq|X|_{t} \leq|X|_{\infty} \in L^{p}
$$

hence $X_{t} \in L_{E}^{p}$. Then for every stopping time $T$ we have

$$
\left|X_{T}\right| \leq|X|_{\infty}
$$


hence $X$ is of class (D). To prove that $I_{X}$ is bounded in $L_{E}^{p}$, let $B \in \mathcal{R}$. Then $B$ is a finite union of disjoint predictable rectangles $B_{0}=\{0\} \times A_{0}$ with $A_{0} \in \mathcal{F}_{0}$, $B_{1}=\left(u_{1}, v_{1}\right] \times A_{1}, \ldots, B_{1}=\left(u_{n}, v_{n}\right] \times A_{n}$ with $A_{i} \in \mathcal{F}_{u_{i}}$ for $1 \leq i \leq n$. We can assume that $0=u_{1}<v_{1}=u_{2}<v_{2}=\ldots=u_{n}<v_{n}$. Then, for each $\varpi \in \Omega$ we have

$$
\begin{aligned}
\left|I_{X}(B)(\varpi)\right| & =\left|\sum_{i} I_{X}\left(B_{i}\right)(\varpi)\right|=\left|\sum_{i}\left(X_{v_{i}}(\varpi)-X_{u_{i}}(\varpi)\right) \mathbf{1}_{A_{i}}(\varpi)\right| \\
& \leq \sum_{i}\left|X_{v_{i}}(\varpi)-X_{u_{i}}(\varpi)\right| \leq|X|_{\infty}(\varpi) .
\end{aligned}
$$

It follows that

$$
\left\|I_{X}(B)\right\|_{p} \leq\left\||X|_{\infty}\right\|_{p}
$$

hence $I_{X}$ is bounded in $L_{E}^{p}$ on $\mathcal{R}$. Now let $E \subset L(F, G)$ be any embedding. Since $X$ has $p$-integrable variation, it has $p$-integrable semivariation relative to $(F, G)$. From the proof of Theorem 6 , we deduce that $I_{X}$ has bounded semivariation relative to $\left(F, L_{G}^{p}\right)$. We can now apply Theorem 3 and deduce that $X$ is $p$-summable relative to $\left(F, L_{G}^{p}\right)$.

If $p=1$ and $X$ has integrable variation, we can remove even the condition $c_{0} \nsubseteq E$.

Theorem 8. Let $X: \mathbb{R}_{+} \times \Omega \longrightarrow E$ be a cadlag, adapted process. If $X$ has integrable variation and if $E \subset L(F, G)$ is any embedding, then $X$ is summable relative to $L\left(F, L_{G}^{p}\right)$.

Proof. We prove as in Theorem 7 that $X$ is of class (D) and that $I_{X}$ is bounded in $L_{E}^{1}$ on $\mathcal{R}$. We prove now that $I_{X}$ has bounded variation. Let $B_{0}, B_{1}, \ldots, B_{n}$ be a family of disjoint sets from $\mathcal{R}$. Without loss of generality, taking $u_{0}=-1, v_{0}=0$, and $\mathcal{F}_{u_{0}}=\mathcal{F}_{0}$, we can assume that $B_{i}=\left(u_{i}, v_{i}\right] \times A_{i}$ with $A_{i} \in \mathcal{F}_{u_{i}}$ for $0 \leq i \leq n$. Then, for every $\varpi \in \Omega$ we have

$$
\begin{aligned}
\sum_{i}\left|I_{X}\left(B_{i}\right)(\varpi)\right| & =\sum_{i}\left|\left(X_{v_{i}}(\varpi)-X_{u_{i}}(\varpi)\right) \mathbf{1}_{A_{i}}(\varpi)\right| \\
& \leq \sum_{i}\left|X_{v_{i}}(\varpi)-X_{u_{i}}(\varpi)\right| \leq|X|_{\infty}(\varpi) .
\end{aligned}
$$

Then

$$
\sum_{i}\left\|I_{X}\left(B_{i}\right)\right\|_{1} \leq\left\||X|_{\infty}\right\|_{1}
$$

Taking the supremum for all the families of sets $B_{i}$ as above, we deduce that

$$
\left|I_{X}\right|\left(\mathbb{R}_{+} \times \Omega\right) \leq\left\||X|_{\infty}\right\|_{1}
$$

hence $I_{X}$ has bounded variation. Then $I_{X}$ is strongly additive ([D, Theorem 6.8]). Let $E \subset L(F, G)$ be any embedding. Then

$$
\left(\widetilde{I_{X}}\right)_{F, G} \leq\left|I_{X}\right|
$$

hence $I_{X}$ has bounded semivariation relative to $\left(F, L_{G}^{1}\right)$. We can apply Theorem $3 \mathrm{~b}$ and deduce that $X$ is summable relative to $\left(F, L_{G}^{1}\right)$.

Remark. This is a new proof of Theorem 19.13b) in [D]. 


\section{REFERENCES}

[D-M] C. Dellacherie and P.-A. Meyer, Probabilités et Potentiel, Hermann, Paris, 1975, 1980. MR0488194 (58:7757) MR0566768 (82b:60001)

[D] N. Dinculeanu, Vector Integration and Stochastic Integration in Banach Spaces, Wiley, New York, 2000. MR1782432 (2001h:60096)

[D-G] N. Dinculeanu and P. Gray, A Local Summability Criterion for Stochastic Integration, J. Ramanujan Math. Soc. 23, No. 1 (2008), 63-76.

[M] M. Métivier, Semimartingales, Walter de Gruyter, Berlin-New York, 1982. MR688144 (84i:60002)

Department of Mathematics, University of Florida, Gainesville, Florida 32611

E-mail address: nd@math.ufl.edu

Department of Mathematics, Lake City Community College, lake City, Florida 32025 